\title{
Cargas de trabalho de enfermeiros: luzes e sombras na Estratégia Saúde da Família
}

\author{
Nurses' workload: lights and shadows in the Family Health Strategy
}

Daiane Biff (https://orcid.org/0000-0002-1118-607X) ${ }^{1}$

Denise Elvira Pires de Pires (https://orcid.org/0000-0002-1754-0922) ${ }^{2}$

Elaine Cristina Novatzki Forte (https://orcid.org/0000-0002-6042-5006) ${ }^{3}$

Letícia de Lima Trindade (https://orcid.org/0000-0002-7119-0230) ${ }^{4}$

Rosani Ramos Machado (https://orcid.org/0000-0001-8287-4171) ${ }^{3}$

Felipa Rafaela Amadigi (https://orcid.org/0000-0003-1480-1231) ${ }^{3}$

Magda Duarte dos Anjos Scherer (https://orcid.org/0000-0002-1465-7949) ${ }^{5}$

Jacks Soratto (https://orcid.org/0000-0002-1339-7268) ${ }^{6}$

${ }^{1}$ Secretaria Municipal de Saúde de Araranguá. R. Cel. Apolinário Pereira 254, Centro. 88900-000 Araranguá SC Brasil. daibiff@hotmail.com ${ }^{2}$ Programa de PósGraduação em Enfermagem. Universidade Federal de Santa Catarina. Florianópolis SC Brasil. ${ }^{3}$ Departamento de Enfermagem. Universidade Federal de Santa Catarina.

Florianópolis SC Brasil.

${ }^{4}$ Programa de Pós-

Graduação em Ciências da Saúde, Universidade Comunitária da Região de Chapecó. Chapecó SC Brasil.

${ }^{5}$ Departamento de Saúde Coletiva. Universidade de Brasília. Brasília DF Brasil. ${ }^{6}$ Programa de PósGraduação em Saúde Coletiva. Universidade do Extremo Sul Catarinense. Criciúma SC Brasil.

\begin{abstract}
This study was multicenter with a qualitative approach, which sought to identify the elements that can increase or reduce the workloads of the Family Health nurse. Forty nurses were interviewed, from 36 teams from five regions of Brazil, considered successful according to the requirements of the National Policy of Primary Care and with good evaluation in the National Program for Improvement in Primary Care Access and Quality. Data collection was performed by instrument triangulation, using a semi-structured interview, observation and documentary study, from 2013 to September 2016. The findings were analyzed based on the Thematic Content Analysis and categorized with the help of Atlas.ti software. It was identified that the elements that most influence the increase of the workloads are the precariousness and deficits in the work environment, materials and equipment, added to the numerical deficit of the workforce and the excess of health care demand. However, teamwork, recognition for the performed work, bonding with users and good interpersonal relationships, all contribute to reduce the workloads. We highlight the dialectic present in the elements of the work process. They can increase or decrease workloads influenced by objective conditions, the moment and way they are handled.
\end{abstract}

Key words Nurses, Work conditions, Work, Primary Health Care, Family Health Strategy
Resumo Estudo multicêntrico, de abordagem qualitativa, que buscou identificar os elementos que podem aumentar ou reduzir as cargas de trabalho do enfermeiro da Saúde da Família. Foram abordados 40 enfermeiros, de 36 equipes, das cinco Regiões do Brasil, consideradas exitosas segundo o prescrito na Política Nacional da Atenção Básica e com boa avaliação no Programa Nacional de Melhoria do Acesso e da Qualidade da Atenção Básica. A coleta de dados foi realizada pela triangulação de instrumentos, utilizando-se entrevista semiestruturada, observação e estudo documental, de 2013 a setembro de 2016. Os achados foram analisados com base na Análise Temática de Conteúdo e categorizados com auxílio do software Atlas.ti. Identificou-se que os elementos que mais influenciam o aumento das cargas são a precariedade e déficits no ambiente de trabalho, materiais e equipamentos, somados ao déficit numérico da força de trabalho e ao excesso de demanda assistencial. Entretanto, o trabalho em equipe, reconhecimento pelo trabalho realizado, vínculo com usuários e bom relacionamento interpessoal contribuem para diminuir as cargas. Ressalta-se a dialética presente nos elementos do processo de trabalho com influência de condições objetivas, do momento e da forma como são manejados.

Palavras-chave Enfermeiros, Condições de Trabalho, Trabalho, Atenção Primária à Saúde, Estratégia Saúde da Família 


\section{Introdução}

A saúde é prioridade na vida das pessoas e integra a agenda internacional do desenvolvimento sustentável formulada pela Organização das $\mathrm{Na}$ ções Unidas ${ }^{1}$, mas a sua garantia como direito universal é um grande desafio ${ }^{2}$.

Nesse cenário assume relevância o debate sobre sistemas de saúde, principalmente em relação ao acesso e melhoria da qualidade do cuidado, com destaque para o financiamento público ${ }^{3} \mathrm{e}$ para o papel desempenhado pela força de trabalho na efetividade dos cuidados de saúde prestados à população. A Organização Mundial da Saúde (OMS) reconhece que "não há saúde sem a força de trabalho"'.

No que diz respeito a quem realiza o trabalho em saúde, há um reconhecimento do papel desempenhado pela enfermagem, tanto pelo contingente numérico, como pela presença em quase todas as instituições de saúde e pelas responsabilidades assumidas no âmbito institucional incluindo ações de cuidado, administrativo-gerenciais e do campo da educação, com forte impacto na qualidade dos serviços prestados ${ }^{4,5}$.

Atualmente a profissão dispõe de $19,3 \mathrm{mi}$ lhões de enfermeiros no mundo ${ }^{6}$. No Brasil, a Enfermagem representa metade da força de trabalho da área da saúde e soma mais de 2 milhões de profissionais, destes, 24\% são Enfermeiros, 56\% técnicos e $20 \%$ auxiliares de enfermagem ${ }^{7,8}$. No que diz respeito ao mercado de trabalho na área da saúde, os enfermeiros constituem a segunda categoria mais expressiva na ocupação dos postos de trabalho de nível superior. Em relação ao nível médio, os técnicos e auxiliares de enfermagem representam mais de $70 \%$ dos postos ocupados na saúde?.

Desde a Conferência Internacional de Alma Ata de 1978, a Atenção Primária à Saúde (APS) tem sido reafirmada como um caminho promissor para o enfrentamento dos graves problemas de saúde que acometem a população do planeta. No Brasil, a APS foi assumida como política governamental integrando a Política Nacional de Atenção Básica (PNAB) no disposto para a Estratégia Saúde da Família (ESF). A enfermagem integra a equipe mínima preconizada pelo Ministério da Saúde e essa presença tem potencial para influenciar a qualidade da assistência prestada à população ${ }^{10}$.

A ESF se propõe a ampliar o acesso aos serviços de saúde, a partir dos princípios que regem o Sistema Único de Saúde no Brasil (SUS). Entretanto, mesmo diante da proposta promissora e dos vários investimentos, há ainda muitos desafios no que se refere a sua efetividade, ressaltando-se a relação público-privado na prestação de serviços de saúde, o subfinanciamento do SUS, a magnitude do país e as intensas desigualdades regionais.

Como outros trabalhos presentes na sociedade atual, a enfermagem sofre influência das condições e relações concretas de trabalho estabelecidas no cotidiano dos cenários de práti$\mathrm{ca}^{11,12}$. Esse cenário tem implicações nas cargas de trabalho (CT) a que os enfermeiros estão expostos diariamente. Estudo realizado por Trindade e Pires ${ }^{13}$ mostra que as condições e relações de trabalho podem interferir de forma negativa nos profissionais da ESF e também no resultado assistencial.

As CT são constituídas por elementos encontrados no processo de trabalho que interatuam entre si e com o corpo do trabalhador podendo gerar desgastes ou adoecimento ${ }^{14}$. Estas são determinadas por fatores que muitas vezes não são claramente identificados pelos próprios profissionais. Conhecer os elementos que contribuem para o aumento e redução das cargas de trabalho colabora para o fortalecimento de aspectos positivos do trabalho e para minimização dos aspectos negativos.

Nesse sentido, este estudo tem como objetivo identificar os elementos que contribuem para a redução e para o aumento das cargas de trabalho de enfermeiros da ESF.

\section{Metodologia}

Estudo de natureza qualitativa, com coleta de dados por meio de triangulação de técnicas (entrevista, observação e estudo documental).

Os participantes foram incluídos pelo critério de intencionalidade: enfermeiros em pleno exercício da função e que estivessem atuando há pelo menos um ano na ESF; que integravam equipes de Saúde da Família (eSF) consideradas de referência (por meio de indicações de gestores e dos resultados da avaliação do primeiro ciclo do Programa Nacional de Melhoria do Acesso e da Qualidade da Atenção básica - PMAQ-AB, publicados em 2013); enfermeiros atuantes em, pelo menos, uma cidade em cada uma das regiões geográficas do Brasil (Florianópolis-SC, região Sul; Rio de Janeiro-RJ, região Sudeste; Natal -RN, região Nordeste; Belém-PA, região Norte e Brasília-DF, região Centro-Oeste); e enfermeiros que estivessem disponíveis na Unidade Básica de 
Saúde (UBS) para entrevista no momento da coleta de dados.

Participaram do estudo 40 enfermeiros de 22 UBS que prestam assistência na modalidade ESF, das cinco Regiões do Brasil, sendo: nove no Sul, nove no Sudeste, sete no Nordeste, seis no Norte e nove no Centro-Oeste.

A Figura 1 mostra o perfil dos participantes da pesquisa segundo características sociodemográficas.

A Figura 2 mostra a caracterização dos participantes em relação a vínculo empregatício e jornada de trabalho.

A coleta ocorreu de março de 2013 a setembro de 2016. Para a realização das entrevistas seguiu-se um roteiro semiestruturado construído com base nas teorias de processo de trabalho Karl Marx ${ }^{11}$ e cargas de trabalho Laurel e Noriega ${ }^{14}$. Além do roteiro de entrevista, foram utilizados roteiros para o estudo documental e observacional, com vistas a entender melhor o contexto onde estão inseridas as eSF.

As observações foram registradas em diário de campo. Os documentos analisados incluíram: planilhas de trabalho e dados do sistema de informação, fichas de produção, atas, livros de registros, painéis e cartazes. Ao final contabilizouse cerca de 40 horas de entrevistas, 196 horas de observação e mais de 300 documentos avaliados.
Os dados foram analisados seguindo os preceitos da análise de conteúdo considerando pré -análise, exploração do material e interpretação e associando-se recursos do software Atlas.ti ${ }^{15}$. Os arquivos de texto foram inseridos no software, realizada a seleção de trechos significativos (quotations) e atribuição de códigos (codes). Os códigos com convergência temática ao objeto de investigação foram aglutinados em grupos (code groups) e por sua vez associados em categorias de análise, apresentadas em redes de visualização (networks).

$\mathrm{O}$ projeto de pesquisa seguiu as exigências das Resoluções 466/2012 e 510/2016 do Conselho Nacional de Saúde ${ }^{16,17}$, tendo sido aprovado pelo Comitê de Ética em Pesquisa com Seres $\mathrm{Hu}$ manos da Universidade Federal de Santa Catarina (UFSC), e pelo Comitê de Ética em Pesquisa com Seres Humanos da Universidade do Estado de Santa Catarina (UDESC).

Os participantes aceitaram fazer parte da coleta de dados e assinaram o Termo de Consentimento Livre e Esclarecido (TCLE). Para garantir o anonimato dos participantes, os mesmos estão codificados conforme sua região de atuação, sendo denominado pela letra E de enfermeiro, região de atuação (S-Sul; N-Norte, SE-Sudeste; NE-Nordeste e CO-Centro-Oeste) e número ordinal da sequência das entrevistas realizadas. Por

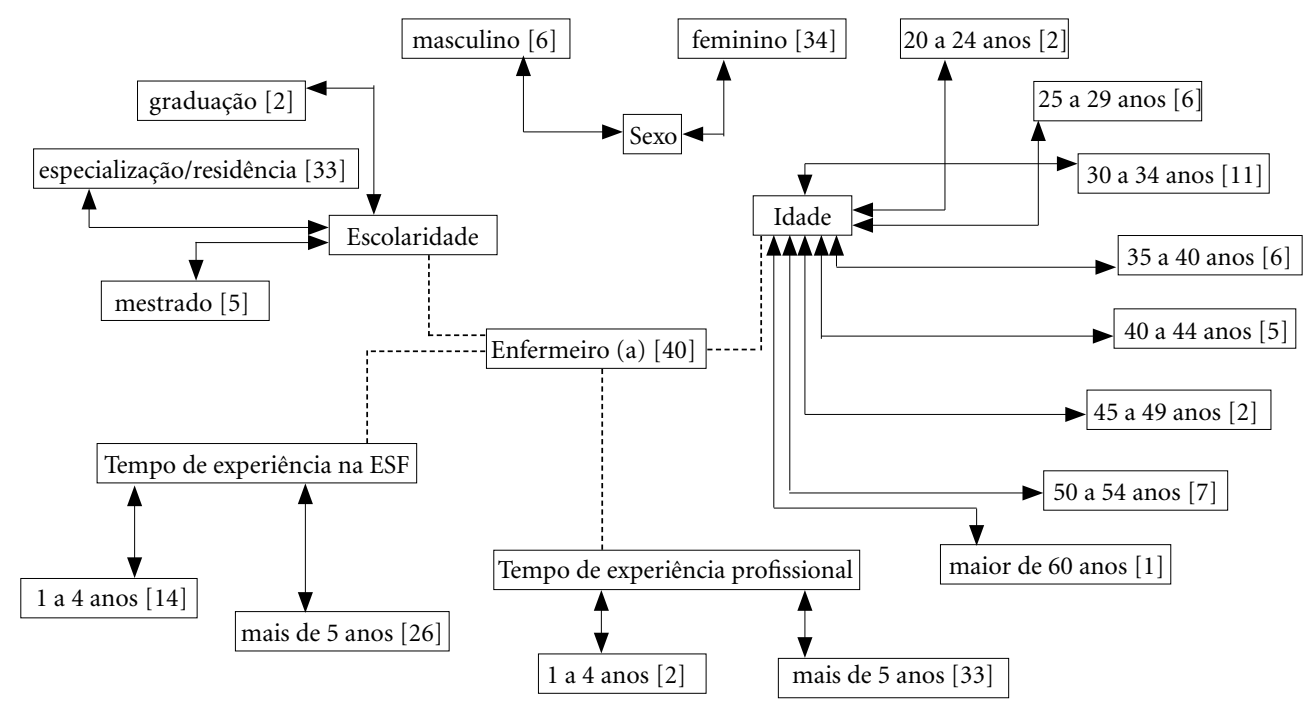

Figura 1. Perfil dos enfermeiros quanto ao sexo, idade, escolaridade, tempo de experiência profissional e na ESF, 2013 a 2016. 


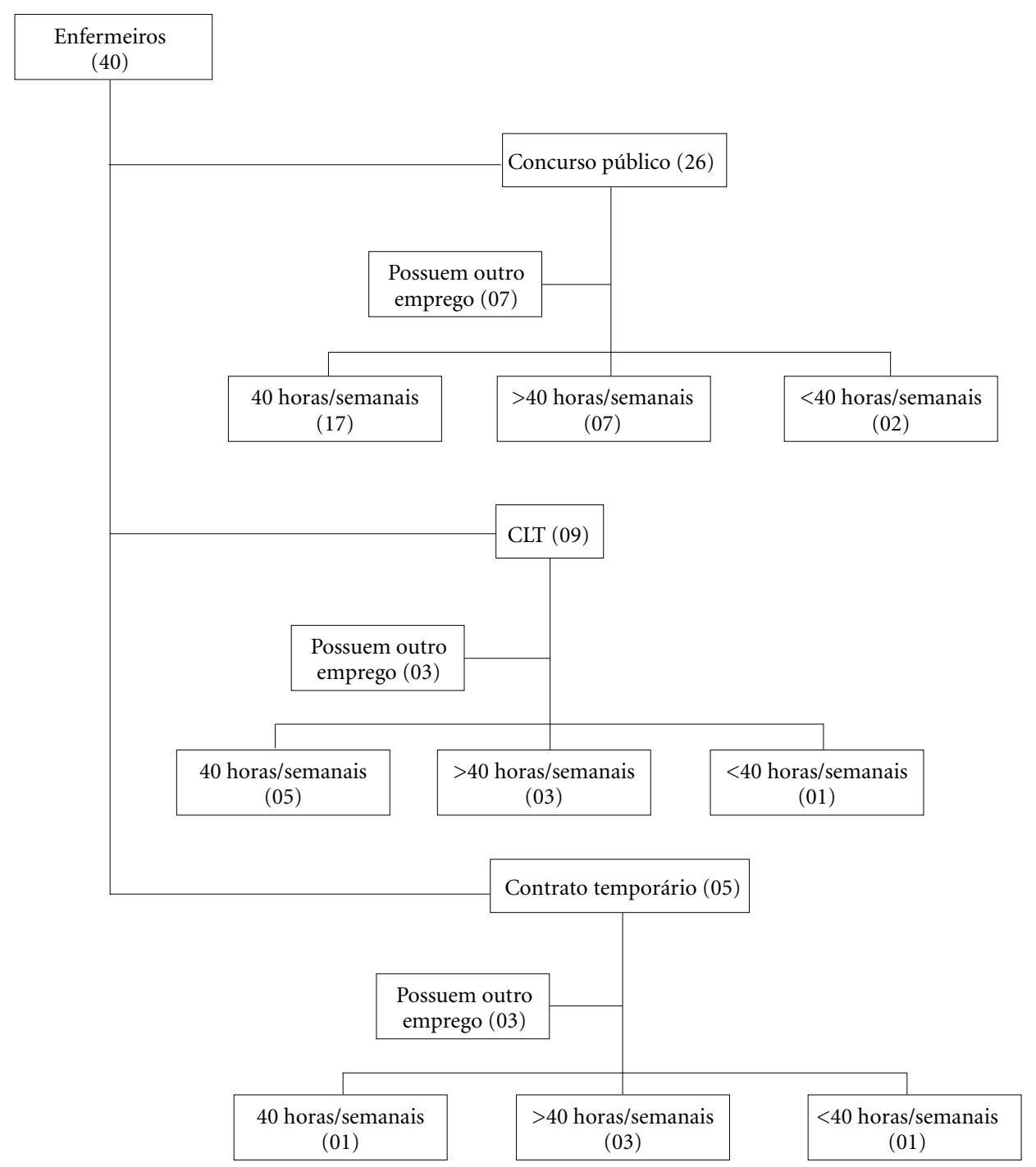

Figura 2. Número de enfermeiros em relação ao vínculo empregatício e jornada de trabalho.

sua vez, para preservar a identificação das UBS observadas utilizou-se a sigla OBS seguida da identificação.

\section{Resultados}

Os resultados foram estruturados em duas macro categorias: elementos presentes no processo de trabalho que contribuem para o aumento e para a redução das CT.

\section{Elementos presentes no processo de trabalho na ESF que contribuem para o aumento das CT dos enfermeiros}

Os principais elementos geradores de CT nos enfermeiros que atuam na ESF estão sintetizados na Figura 3.

Dentre todos os elementos presentes no processo de trabalho que contribuem para aumentar as CT, destacou-se a precariedade do ambiente de trabalho.

Um desgaste [...] eu cheguei aqui, algumas coisas já me irritavam muito [...] "não tem consultó- 


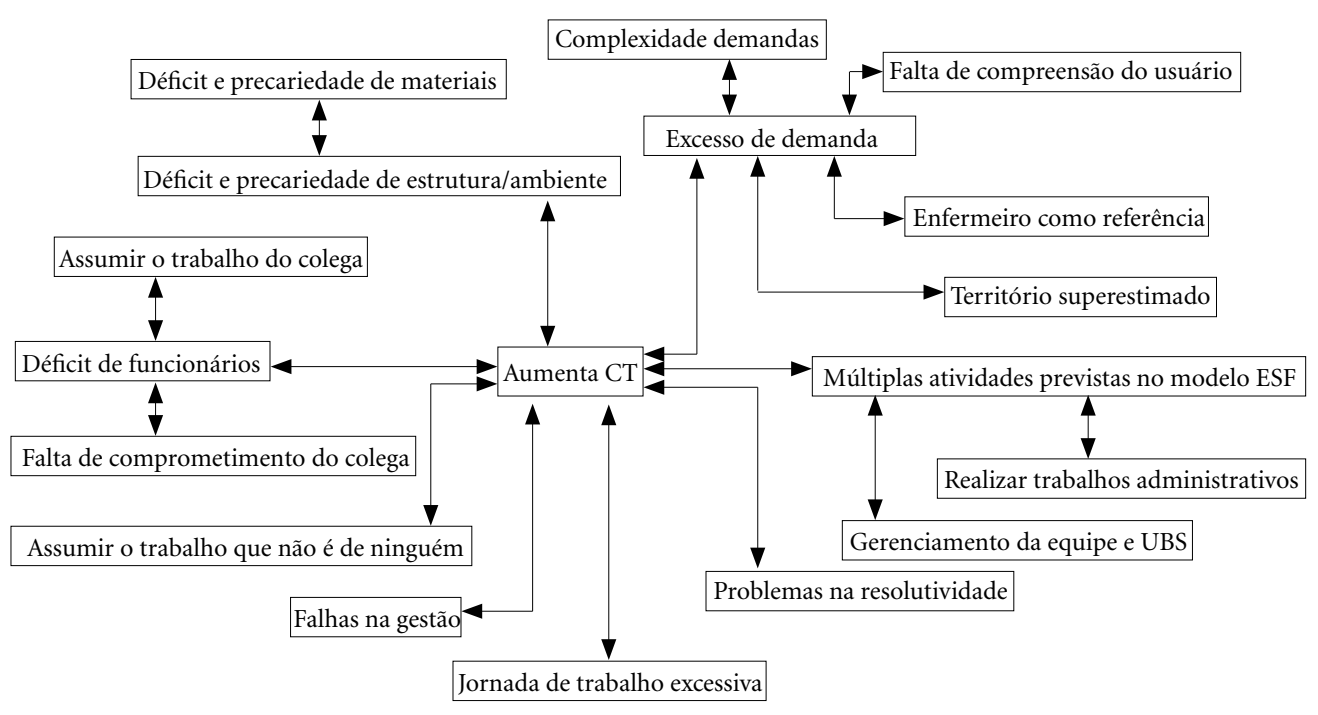

Figura 3. Elementos que contribuem para aumentar as cargas de trabalho dos enfermeiros atuantes nas ESF. Brasil, 2013-2016.

rio". Um desocupa e você fala "posso atender uma pessoa aqui?" Aí você entra e o outro fica aguardando (ESE1).

A escuta feita pelos profissionais aos usuários acontece na recepção, nos corredores ou em consultório, caso esteja disponível, e dependendo do teor das informações (OBS Nordeste).

A estrutura física é muito deficiente, não temos onde colocar muitas coisas. E você pode ver que aqui era para ser um auditório, acabou virando um consultório. Foram só multiplicando as equipes e não foi pensado em montar uma estrutura física adequada (ECO7).

A falta ou precariedade de materiais e instrumentos pode influenciar negativamente no cuidado, gerar retrabalho, assim como desgaste do trabalhador e das relações com os usuários.

Tem gente que teve que mandar paciente embora. Eu tinha quatro lâminas, os primeiros que chegarem vou colher, os outros vão ter que ir embora e remarcar para outro dia porque você não tinha materiais para fazer [...]. Eu acho que a primeira coisa é a falta de recursos [...], isso aumenta bastante, tanto a carga emocional, quanto de trabalho mesmo (ECO4).

Estou com uma autoclave estragada há mais de 1 ano e para conseguir a troca dessa autoclave é uma demora, pois depende de abertura de licitação para comprar uma nova, o que faz com que eu tenha que esperar pela solução (ES2).

Outro importante elemento causador do aumento das CT é o excesso de demanda nas UBS, aliada à complexidade das necessidades em saúde e ao contingente significativo de usuários que vivem em situação de vulnerabilidade social. Além disso, também aumenta as CT, atuar não apenas dentro do espaço físico da UBS, mas também no âmbito da comunidade, de forma a atender uma das prerrogativas da ESF. Associa-se a este cenário a existência de territórios com populações subestimadas.

Aumenta muito a carga porque o número de atendimentos é muito grande, acaba que deixa de ver algumas outras coisas que tinha que ver, e assim, a gente não vai conseguir nenhuma qualidade (ESE9).

Um dos principais problemas é a quantidade de demanda. Um território contém mais de $40 \mathrm{mil}$ habitantes para uma UBS que dispõe de apenas 7 equipes de saúde da família e 3 equipes de Saúde Bucal. Com vários profissionais ausentes por férias, afastamentos, entre outros (OBS Sul).

Os enfermeiros relatam a questão do déficit de funcionários como gerador de aumento das 
suas CT. O que se agrava pela falta de comprometimento de alguns profissionais que deixam de realizar as atividades de sua competência por falta ao trabalho, negligência ou desmotivação, o que leva alguns enfermeiros a assumirem o trabalho dos colegas e aquele trabalho que "não é de ninguém".

A gente vai ficar seis, sete meses sem estes profissionais (uma enfermeira e um dentista), pois ele (dentista) ainda tem férias ou alguma licença-prêmio que tem de direito. E aí sobrecarrega quem está na unidade uma vez que essa área não vai desaparecer porque o profissional não está. Pelo contrário, [os que moram nesta área] vem e a gente tem que diluir entre quem está na unidade (ES8).

Eu levo muito trabalho pra casa, porque aqui tenho muitas atribuições, além de ter que fazer visita, de atender o pré-natal, ter que colher preventivo, tratar do diabético, do hipertenso, fazer as orientações coletivas, participar do programa saúde na escola, tem a população que quer atendimento para a demanda espontânea, para a emergência. Acho isso muito complicado, essas tantas atribuições (ECO2).

O excesso de atribuições, incluindo as que não lhe competem como, por exemplo, atividades burocráticas existentes mesmo em UBS com gestor contratado para esta função, aliado às atividades assistenciais e educacionais implicam tanto no trabalho quanto na saúde dos enfermeiros.

[...] pois é, liga dali, liga para cá, resolve ali e mais as questões mesmo de atendimento de enfermagem. [...] Eu sinto que os enfermeiros são muito sobrecarregados na atenção básica (ESE3).

Os profissionais consideram, nesse contexto, que a carga horária de trabalho é muito elevada, devido à intensidade da jornada, como explica a participante:

A carga horária é muito pesada. E isso aumenta a minha ansiedade, já tive pico de estresse, já tive pico de ansiedade (ESE5).

As falhas na gestão, tanto municipal quanto no âmbito da APS, estão entre os motivos relevantes de aumento das CT. As falhas estão relacionadas à falta de profissionais nas equipes, às cobranças no trabalho, consideradas, muitas vezes, desnecessárias, mas também à ausência de atuação do gestor.

A coordenação geral, eu acho que nem sempre ela facilita o meu trabalho, que eu posso considerar sobrecarga porque vai me sobrecarregar depois, algumas solicitações da coordenação e da CAPP [Coordenação de Área Planejada e Programada] para mim elas não colaboram com o meu processo de trabalho aqui (ESE4).

Você tem que parar para resolver uma coisa que uma gestão mal formada cria [...] era justamente para ser o contrário. Eu acho que o gestor está ali para te ajudar e não para te criar problema (ECO7).

Os enfermeiros relataram a angústia de tentar realizar todas as tarefas necessárias e ter dificuldades em realizar o solicitado e o que consideram importante.

A minha auto avaliação é que eu não dou conta. Eu não dou conta de fazer o atendimento, preencher o meu eSUS, digitar o meu eSUS, dar conta das demandas que o enfermeiro tem de responder ao Distrito, de fazer a planilha das ações educativas. Então existe uma tristeza por não dar conta, isso tudo gera um sofrimento (END7).

O trabalho do enfermeiro na estratégia inclui bastante atividade burocrática, de gestão por conta de tudo. Como o volume de atendimentos também é muito grande, às vezes eu sinto que eu não estou cumprindo todas as atividades que eu preciso (ESE4).

\section{Elementos presentes no processo de trabalho na ESF que contribuem para reduzir as $\mathrm{CT}$ dos enfermeiros}

O trabalho na ESF pode ser gerador de estresse e adoecimento, entretanto, existem elementos que contribuem para que este mesmo trabalho se torne menos difícil, ou até mesmo mais prazeroso, com impacto positivo nas CT. A síntese destes achados está apresentada na Figura 4.

Os enfermeiros mencionaram, com maior ênfase, o trabalho em equipe como principal responsável por reduzir as CT. O trabalho colaborativo em equipe, com comprometimento do conjunto dos participantes e planejamento das ações contribui para o bom funcionamento do trabalho na ESF, tornando-o mais eficaz e prazeroso.

Saber que a gente tem uma equipe que trabalha e pensa realmente no usuário facilita [...] traz segurança até para a própria equipe trabalhar [...] [contar com] o comprometimento de todos os funcionários (ESE7).

Na minha unidade eu tenho uma equipe muito boa e a gente tem uma interação muito bacana. Então isso ajuda muito para poder realizar tudo (EN4).

A gente sempre traz tudo para a reunião de equipe para dividir, porque é uma coisa muito importante. [...] não tem uma decisão que não seja tomada por todos, todas são discutidas [...] [para] ver a melhor maneira para aquilo (ESE9).

Nesse contexto também fica claro que apenas a equipe mínima prescrita pela Política Nacional da Atenção Básica (PNAB) não é suficiente para conciliar todas as exigências prescritas. 


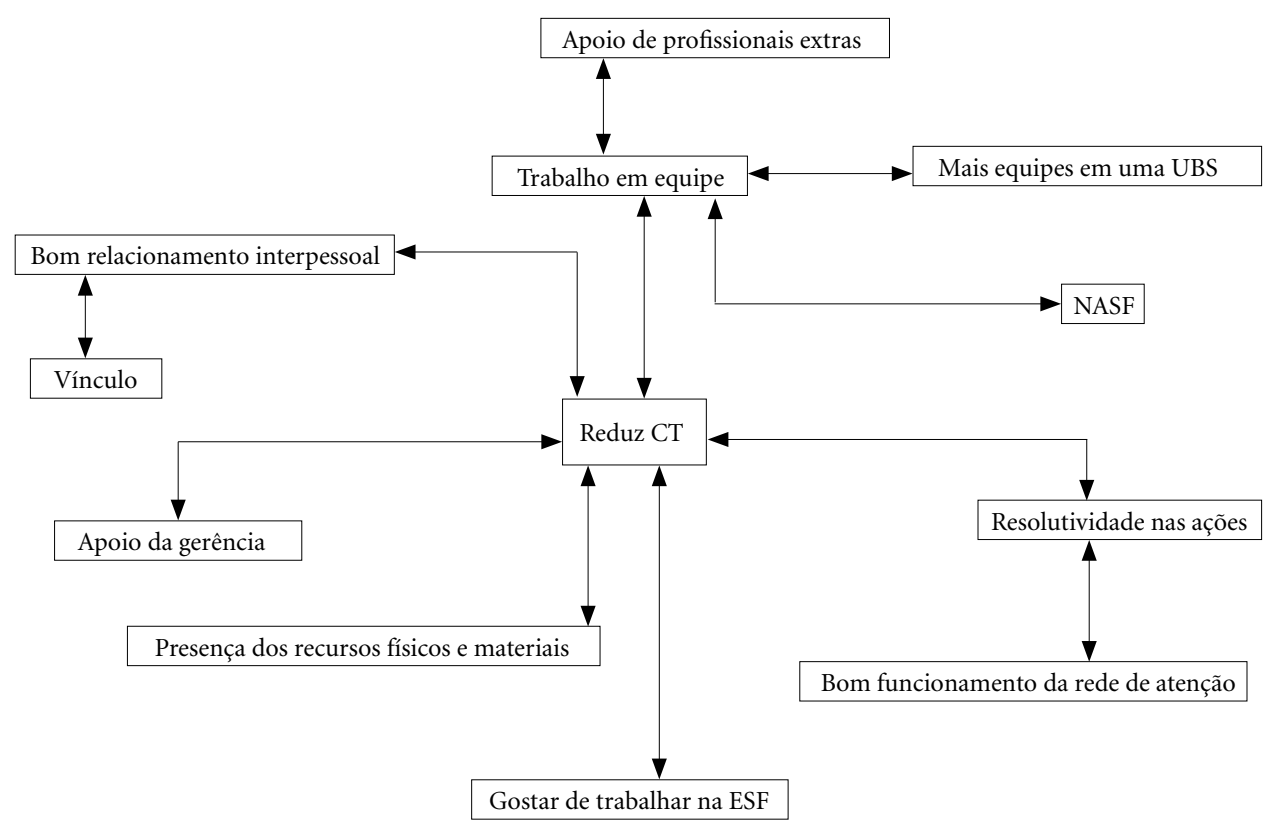

Figura 4. Elementos do trabalho na ESF que contribuem para reduzir as cargas de trabalho dos enfermeiros. Brasil, 2013-2016.

A gente tem o NASF que é um Núcleo de Apoio à Saúde da Família e o psicólogo participa muito das conversas da gente, a nutricionista se envolve também em alguns casos. Então, participar [da assistência] do paciente de uma forma integral é bacana (ESE8).

Para que todos os trabalhadores consigam trabalhar em equipe e efetuar suas tarefas adequadamente, o bom relacionamento interpessoal aliado a uma boa comunicação são elementos necessários no trabalho em saúde.

A gente tem uma relação boa, de troca mesmo, a relação é muito boa (ENE7).

O compromisso de todo mundo aqui dentro me motiva. Todos sabem das suas responsabilidades $e$ cada um se preocupa com o bem do próximo, em se colocar no lugar do outro. Isso me motiva a continuar aqui (ECO2).

Vínculo positivo com o usuário contribui para que ele se sinta parte do processo, facilitando a ação terapêutica e respondendo melhor aos tratamentos e orientações.

A vinculação é muito boa, o fato de conhecer os pacientes, isso ajuda muito. Você sabe qual é o paciente, que ele pode esperar [...] pode pedir para ele voltar à tarde ou não (ECO7).
[...] É uma população que compreende se a gente diz um não, dificilmente a gente tem um problema maior, e isso se deve ao vínculo (ES8).

$\mathrm{O}$ funcionamento adequado das redes de atenção e a resolutividade nas ações influenciam positivamente na qualidade da assistência prestada e na satisfação de usuários e profissionais, contribuindo para a redução das CT.

Uma gestante precisa de uma avaliação com a nutricionista, eu consigo aqui, não precisa encaminhar. Então eu facilito para mim, facilito para o usuário, que não precisa ficar se deslocando, não é? (ESE7).

A gente conseguir resolver [...] perceber que de fato, a gente conseguiu. Nosso objetivo maior que é, na verdade, o paciente, é a melhoria dele (ECO8).

Quando o enfermeiro se sente parte do processo de trabalho e "gosta do que faz", o fazer diário fica mais "leve" e, aparentemente, mais simples.

Eu gosto muito do que eu faço, eu estudo todos os dias (ES4).

Gostar de estar na unidade. Eu sinto falta. O dia em que eu não estou aqui eu ligo, eu acompanho e-mail, acompanho whatsapp (ESE7).

Para que o enfermeiro consiga realizar um trabalho de qualidade, é necessário existir apoio 
de colegas, usuários e das gerências de todas as instâncias governamentais, sendo esses apoios importantes na redução das CT. O gestor deve ser aquele que facilita o processo e contribui para $\mathrm{o}$ planejamento e organização do trabalho.

A gente poder conversar e reajustar os nossos atendimentos com o que a gente tem. Isso dá um certo alívio, da gente ter um apoio da gestão para poder agir dessa maneira. Ter uma liberdade de implementar os nossos atendimentos conforme o que a gente tem. E isso traz conforto (ECO9).

E o apoio da gerência de forma geral, da administração, eles nos apoiam muito, assim. Isso é muito bom para gente [...], saber que a gente tem esse apoio da administração, não só da gerência, da administração da clínica (ESE5).

Além dos elementos ligados à força de trabalho, ao objeto de trabalho e às relações que permeiam o processo de trabalho, existem os elementos relacionados aos instrumentos. Estes, quando presentes em quantidade e qualidade adequadas, tornam-se aliados e contribuem para facilitar o processo assistencial e gerencial.

Acho que a estrutura da unidade, apesar de termos aumentado o número das equipes e não termos consultórios suficientes para todo mundo, a gente se vira. E em questão de material, nós temos suficiente na medida do possivel, então ainda não faltou totalmente alguma coisa que não consigamos trabathar, e isso é importante ter material para trabalhar (ES7).

\section{Discussão}

$\mathrm{Na}$ atualidade brasileira, a ESF constitui-se em importante mercado de trabalho dos enfermei$\operatorname{ros}^{18} \mathrm{e}$ o estudo do trabalho destes profissionais, em especial dos aspectos/elementos presentes no seu processo de trabalho que podem influenciar positiva ou negativamente nas suas cargas de trabalho, assume relevância significativa.

No que diz respeito ao perfil sociodemográfico dos enfermeiros participantes deste estudo houve predominância de mulheres, adultas jovens, com significativo tempo de experiência $(82,5 \%$ com mais de 5 anos) e boa qualificação, sendo que $82,5 \%$ concluiu cursos de Residência ou de Especialização.

Os achados se aproximam da pesquisa do perfil da enfermagem no Brasil, no que diz respeito à hegemonia feminina e a predominância de jovens na profissão $0^{7,8}$. Da mesma forma, em relação à qualificação, estudos anteriores realizados nas regiões Centro-Oeste, Norte e Sudeste do Brasil ob- tiveram as porcentagens de $53,1 \%, 80 \%$ e $93,8 \%$, respectivamente, de enfermeiros atuantes na ESF com pós-graduação $0^{19-21}$.

Equipes de ESF que possuem enfermeiros com maior experiência e maior grau de escolaridade apresentam resultados mais satisfatórios em relação às exigências do modelo de atenção tais como a acessibilidade, a longitudinalidade, a capacidade de coordenação e o atendimento integral às famílias e comunidade ${ }^{21,22}$. A partir do prescrito na $\mathrm{PNAB}^{10}$, atribui-se ao profissional enfermeiro a realização de uma assistência integral, que abrange a promoção e a proteção da saúde, prevenção de agravos, tratamento, reabilitação e manutenção da saúde de indivíduos e famílias. Melhor formação contribui para atingir a eficiência, a eficácia e a efetividade almejadas para a consecução da atenção integral.

Dentre os elementos que aumentam as cargas na ESF destaca-se a precariedade do ambiente de trabalho, relacionada a deficiências na estrutura física e falta de materiais, ou seja, déficits e carências nos instrumentos de trabalho. Na literatura também há registro de que a carência de equipamentos e insumos está presente na maioria das UBS do Brasil, restringindo o escopo e a resolutividade das ações, e limitando a capacidade de resposta aos problemas de saúde da população $0^{23}$. Instrumentos de trabalho, em sentido ampliado ${ }^{11}$, incluem o que é colocado entre o trabalhador $\mathrm{e}$ o que será por ele transformado e, ainda, o ambiente onde o trabalho se realiza. Déficits e precariedade dos mesmos dificultam a realização do trabalho e geram aumento de cargas. Trata-se de uma problemática cuja resolução foge do espaço de governabilidade dos profissionais, e que ao limitar o agir traz sentimentos de tristeza e impotência, gerando desmotivação e insatisfação, conforme revelado pelos enfermeiros deste estudo.

O excesso de demanda associado aos déficits no quantitativo da força de trabalho emergiram como elementos marcantes no aumento das cargas de trabalho. Estes dois aspectos articulam: o acúmulo de funções que o enfermeiro exerce além das assistenciais, muitas vezes sendo referência para o funcionamento da UBS; o território superestimado e o déficit de força de trabalho, relacionado ao ter que assumir o trabalho de colegas; a complexidade das demandas de saúde da população e as dificuldades em atender as expectativas dos usuários. Todos estão incluídos no macro conceito de condições de trabalho, destacando-se que "o como" se realiza determinada atividade é fundamental na análise de qualquer processo de trabalho ${ }^{11}$. 
O enfermeiro assume a coordenação do trabalho da enfermagem e, muitas vezes, da UBS. Assim, amplia sua atuação agregando atribuições para além do central de seu núcleo profissional. Apesar da relevância da gestão da UBS para a atenção à saúde ${ }^{24}$, a duplicidade de atividades gera aumento das cargas de trabalho. $\mathrm{O}$ excessivo número de atividades desenvolvidas pelos enfermeiros pode desencadear uma série de processos que envolvem frustração e estresse, sendo que trabalhadores estressados estão mais suscetíveis aos acidentes de trabalho e às doenças ocupacionais de cunho emocional, como a depressão, a ansiedade e padrão de sono alterado, que caracterizam a diminuição da produtividade e os altos índices de absenteísmo ${ }^{25}$. As demandas excessivas constituem um estressor que torna os trabalhadores mais desmotivados ${ }^{25}$. Somam-se a esse quadro as possíveis repercussões na qualidade dos cuidados prestados aos usuários, em termos inclusive de segurança dos pacientes ${ }^{26}$.

Ainda foram geradores de aumento das CT problemas na gestão, os quais interferem na resolutividade da assistência, nos modos de organização e gestão do trabalho e em aspectos das condições de trabalho como a jornada de trabalho excessiva. $\mathrm{A} \mathrm{PNAB}^{10}$ prevê a obrigatoriedade de carga horária de 40 horas semanais para enfermeiros e outros membros da eSF, o que correspondeu ao encontrado na pesquisa: maioria significativa dos enfermeiros participantes do estudo tem jornada de trabalho de 40 horas semanais ou mais.

No cenário recente da atenção à saúde no Brasil soma-se outras "sombras", com possíveis repercussões no mercado e nas condições de trabalho dos enfermeiros e demais trabalhadores e impacto negativo na APS/ESF. Destaca-se a Emenda Constitucional no 95, aprovada em $2016^{27}$, que congelou os gastos públicos por 20 anos, afetando sobremaneira o SUS, historicamente subfinanciado. Ou seja, poderá haver corte nos contratos de trabalho e os profissionais precisarão atender uma população crescente dispondo dos mesmos recursos. Nesse cenário crescem as exigências de aumento da produtividade, levando à imposição de ritmos e rupturas que se afastam do tempo da vida humana, exigindo adaptabilidade e flexibilidade $^{28}$. O contexto de escassez de recursos pode gerar intensificação de cobranças por parte dos gestores contribuindo para o aumento das CT.

Também se destaca como "sombra" à efetividade da APS, as mudanças constantes na PNAB como a aprovada em $2017^{10}$, que alteram aspectos fundamentais nos atributos da APS, tais como a longitudinalidade do cuidado.
Por outro lado, identificaram-se elementos no processo de trabalho dos enfermeiros que reduzem as cargas de trabalho, sendo o trabalho em equipe um dos mais significativos. A cooperação, colaboração e divisão de responsabilidades no trabalho em equipe contribuem consideravelmente na melhoria do processo de trabalho e estimulam os profissionais a enfrentar suas dificuldades a partir das especificidades de cada um e do respeito às diversas formas de lidar com os desafios. Esse achado reporta ao reconhecimento de que os modos de organização e relações impactam na força de trabalho ${ }^{11}$, em especial na saúde dos trabalhadores ${ }^{14}$.

No trabalho em equipe, os resultados obtidos são maiores do que a soma dos resultados individuais, aumentando a eficácia e a eficiência do atendimento prestado à população $0^{29}$. Trabalho em equipe não significa trabalhar sempre de forma harmoniosa, o diferencial está em converter os conflitos em crescimento, sabendo trabalhar com diferenças de ideias ou de condutas e, portanto, agir profissionalmente na presença de conflitos $^{30}$. Assim, o bom relacionamento emergiu como redutor das cargas de trabalho e mediador das dificuldades no trabalho dos participantes.

A proposta do NASF fortalece o trabalho em equipe, pois também envolve a corresponsabilização e a gestão do cuidado integrada com os profissionais das equipes de Saúde da Família (eSF). A assistência e os projetos terapêuticos são compartilhados e contribuem para melhorar a resolutividade das ações. Esse apoio matricial também foi mencionado como positivo em outro estudo ${ }^{31}$, considerando-o como suporte às equipes para a resolução dos problemas, prestando assistência aos usuários e minimizando a demanda por atendimento especializado.

A afinidade com o trabalho e o vínculo contribuem para reduzir as cargas de trabalho. A afinidade possibilita olhar para o trabalho e verse como parte integrante, e importante, para a obtenção dos resultados, o que contribui para a redução das CT. A importância do vínculo na Saúde da Família está registrado na literatura recente $^{32}$. Este possibilita que o enfermeiro se torne referência na assistência aos usuários, criando laços de cumplicidade entre profissional e usuário, e tem sido visto como facilitador das práticas na ESF, contribuindo para a produção de um cuidado mais eficaz ${ }^{33}$.

Boas condições de trabalho, incluindo o bom funcionamento da rede de atenção, a resolutividade da assistência e o apoio gerencial também foram mencionados como significativos para a 
redução das CT dos enfermeiros. Condições, organização e relações de trabalho, como menciona a teoria de processo de trabalho ${ }^{11}$ e de $\operatorname{cargas}^{14}$, dialeticamente, geram aumento das CT quando deficitárias e redução quando satisfatórias.

Além de serem facilitadores do trabalho, o funcionamento adequado das redes de atenção e a resolutividade nas ações influenciam positivamente na formação do vínculo com os usuários. Poder contar com outros pontos de atenção à saúde e saber que estes realizarão seu papel de forma eficaz e segura, contribui para a consolidação da confiança entre profissionais e usuários e garante a qualidade e continuidade do cuidado.

Em síntese, os achados deste estudo aproximam-se do encontrado por pesquisadores no tema $^{12,13}$, ao estudarem o conjunto dos profissionais que atuam nas eSF. Os referidos estudos descrevem que os principais elementos geradores de aumento das CT nas equipes multiprofissionais da ESF estão relacionados com as condições de trabalho, como: sobrecarga; excesso de demanda; déficits na estrutura física; disponibilidade de força de trabalho insuficiente; salários considerados insuficientes e jornada de trabalho excessiva. Todos esses elementos possuem forte relação com a gestão dos serviços. As autoras ${ }^{12,13}$ referem, ainda, que o trabalho em equipe, o vínculo com os usuários e a afinidade com o trabalho realizado são fatores que diminuem as cargas, constituindo-se em aspectos protetores do trabalho.

\section{Considerações finais}

O estudo permitiu identificar os elementos do processo de trabalho de enfermeiros da ESF que contribuem para a diminuição e aumento das cargas de trabalho. No que diz respeito ao processo de trabalho se destacaram elementos relacionados às condições e relações de trabalho, a gestão do trabalho e ao objeto de trabalho das enfermeiras e profissionais das eSF (que são usuários do serviço). O estudo identificou problemas nas condições de trabalho na ESF, nas cinco Regiões no país, interferindo na prática profissional dos enfermeiros.

As CT são intensificadas pelos déficits, ainda existentes, na estrutura física e nos recursos humanos e materiais, pela alta demanda de trabalho exigida e pelos problemas relacionados com a gestão das unidades e dos municípios. $\mathrm{O}$ aumento das CT pode gerar insatisfação, desgaste e, até, adoecimento nestes profissionais.

Se há sombras, também há luzes, e nesse sentido os enfermeiros abrigam-se à luz do trabalho em equipe, no gostar do que fazem, no apoio por parte das equipes do NASF e nos bons relacionamentos com os usuários e colegas de trabalho. Assim motivam-se para exercerem suas funções cotidianas, com vistas à continuidade e qualidade do cuidado.

Ressalta-se nesse estudo a dialética presente nos elementos do processo de trabalho que podem aumentar ou diminuir as cargas de trabalho. A influência positiva ou negativa dessa relação depende de condições concretas, objetivas, mas também do momento e da forma como esses elementos se manifestam, e de como são manejados pelos enfermeiros.

Os resultados do estudo fornecem subsídios para compreender como as cargas de trabalho se expressam nesse importante mercado de trabalho da enfermagem, na atualidade no Brasil. Contudo, cabe ressaltar que a pesquisa envolveu somente equipes de Saúde da Família consideradas de referência para os gestores locais e que, na maioria, tiveram boa avaliação do PMAQ-AB. Assim outras investigações em realidades diferentes devem ser realizadas, com vistas a contribuir para ampliar o conhecimento sobre as cargas de trabalho presentes na APS, bem como fornecer subsídios aos formuladores de políticas públicas, no sentido de repensar o processo de trabalho nesse contexto, em benefício das diretrizes e princípios do SUS e do acesso universal. 


\section{Colaboradores}

D Biff, DEP Pires e ECN Forte trabalharam na concepção, delineamento do estudo, análise e interpretação dos dados. D Biff, MDA Scherer e J Soratto participaram na coleta de dados. D Biff, DEP Pires, ECN Forte, LL Trindade, RR Machado, FR Amadigi, MDA Scherer e J Soratto colaboraram na redação e revisão crítica do artigo.

\section{Agradecimentos}

Ao apoio e financiamento do Conselho Nacional de Desenvolvimento Científico e Tecnológico CNPq.

\section{Referências}

1. Buss PM, Fonseca LE, Galvão LAC, Fortune K, Cook C. Health in all policies in the partnership for sustainable development. Rev Panam Salud Publica 2016; 40(3):186-191.

2. Giovanella L, Mendoza-Ruiz A, Pilar ACA, Rosa MC, Martins GB, Santos IS, Silva DB, Vieira JML, Castro VCG, Silva PO, Machado CV. Sistema universal de saúde e cobertura universal: desvendando pressupostos e estratégias. Cien Saude Colet 2018; 23(6):17631776.

3. Viacava F, Oliveira RAD, Carvalho CC, Laguardia J, Bellido JG. SUS: oferta, acesso e utilização de serviços de saúde nos últimos 30 anos. Cien Saude Colet 2018; 23(6):1751-1762.

4. World Health Organization (WHO). Global Health Workforce Alliance [página na Internet]. 2013 [acessado 2019 Fev 18]. Disponível em: http://www.who.int/ workforcealliance/en/

5. Pires DEP. A enfermagem enquanto disciplina, profissão e trabalho. Rev Bras Enferm 2009; 62(5):739-744.

6. Mendes IAC, Ventura CAA. Nursing Protagonism in the UN Goals for the people's health. Rev Lat Am Enfermagem [periódico na Internet]. 2017 [acessado 2019 Fev 21]; 25:e2864. Disponível em: http://www. scielo.br/pdf/rlae/v25/pt_0104-1169-rlae-25-02864. pdf

7. Machado MH, Aguiar Filho W, Lacerda WF, Oliveira E, Lemos W, Wermelinger M, Vieira M, Santos MR, Souza Junior PB, Justino E, Barbosa C. Características gerais da enfermagem: o perfil sócio demográfico. Enferm Foco 2016; 7(n. esp.):9-14.

8. Conselho Federal de Enfermagem (COFEN). Enfermagem em números [página na Internet]. 2019 [acessado 2019 Fev 21]. Disponível em: http://www.cofen. gov.br/enfermagem-em-numeros

9. Instituto Brasileiro de Geografia e Estatística (IBGE). Estatísticas da Saúde: assistência médico-sanitária 2009 [documento na Internet]. Rio de Janeiro: IBGE; 2010. [acessado 2019 Fev 15]. Disponível em: https://biblioteca.ibge.gov.br/visualizacao/livros/liv46754.pdf

10. Brasil. Portaria no 2.436, de 21 de setembro de 2017. Aprova a Política Nacional de Atenção Básica, estabelecendo a revisão de diretrizes para a organização da Atenção Básica, no âmbito do Sistema Único de Saúde (SUS). Diário Oficial da União 2017; 22 set.

11. Marx K. O capital: crítica da economia política. Livro I. $3^{\text {a }}$ ed. Rio de Janeiro: Civilização Brasileira; 2012.

12. Pires DEP, Machado RR, Soratto J, Scherer MA, Gonçalves ASR, Trindade LL. Cargas de trabalho da enfermagem na saúde da família: implicações no acesso universal. Rev Lat Am Enfermagem [periódico na Internet]. 2016 [acessado 2019 Fev 15]; 24:e2682. Disponível em: 10.1590/1518-8345.0992.2682.

13. Trindade LL, Pires DEP. Implicações dos modelos assistenciais da atenção básica nas cargas de trabalho dos profissionais de saúde. Texto Contexto Enferm 2013; 22(1):36-42.

14. Laurel AC, Noriega M. Processo de produção e saúde: trabalho e desgaste operário. São Paulo: Hucitec; 1989.

15. Friese S, Soratto J, Pires DEP. Carrying out a computer -aided thematic content analysis with ATLAS.ti. $M a$ $x$-Planck-Institut zur Erforschung multireligiöser und multiethnischer Gesellschaften 2018; 2:1-30. 
16. Brasil. Resolução n ${ }^{\circ}$ 446, de 12 de dezembro de 2012. Normas regulamentadoras de pesquisas envolvendo seres humanos. Diário Oficial da União 2013; 13 jun.

17. Brasil. Resolução n ${ }^{\circ}$ 510, de 07 de abril de 2016. Normas aplicáveis a pesquisas em Ciências Humanas e Sociais. Diário Oficial da União 2016; 24 maio.

18. Oliveira JSA, Pires DEP, Alvarez AM, Sena RR, Medeiros SM, Andrade SR. Tendências do mercado de trabalho de enfermeiros/as na visão de gestores. Rev Bras Enferm 2018; 71(1):148-155.

19. Santana FR, Santana FR, Anjos GV, Campos TV, Lima PCT, Lopes MM, Lima RP, Oliveira NS, Weirich CF, Fortuna CM. Ações de saúde na estratégia saúde da família no município goiano na perspectiva da integralidade. Rev Eletr Enf 2013; 15:422-429.

20. Rocha ACD, Sousa CPC, Queiroz D, Pedraza DF. Atenção básica à saúde: avaliação de estrutura e processo. Rev Adm Saude 2012; 14(54):71-79.

21. Lima EFA, Primo CC, Leite FMC, Souza MHN, Maciel EEN. Perfil socioprofissional de trabalhadores de equipes saúde da família. Rev Enferm UERJ [periódico na Internet]. 2016 [acessado 2019 Fev 02]; 24(1):e9405. Disponível em: https://doi.org/10.12957/ reuerj.2016.9405

22. Silva LMS, Fernandes MC, Mendes EP, Evangelista NC, Torres RAM. Trabalho interdisciplinar na Estratégia Saúde da Família: enfoque nas ações de cuidado e gerência. Rev Enferm UERJ 2012; 20(2):784-788.

23. Bousquat A, Giovanella L, Fausto MCR, Fusaro ER, Mendonça MHM, Gagno J, d'Ávila Viana AL. Tipologia da estrutura das unidades básicas de saúde brasileiras: os 5 R. Cad Saude Publica [periódico na Internet]. 2017 [acessado $2019 \mathrm{Fev} 23$ ]; 33(8):e00037316. Disponível em: http://dx.doi.org/10.1590/0102-311x 00037316

24. Melo RC, Machado ME. Coordenação de unidades de saúde da família por enfermeiros: desafios e potencialidades. Rev Gauch Enferm 2013; 34(4):61-67.

25. Rosario CAR, Lopes AM, Pereira FFA, Costa FM. Avaliação do estresse entre enfermeiros que atuam na Estratégia Saúde da Família de Montes Claros, MG. Renome 2015; 4(1):3-14.

26. Magalhães AMM, Dall'Agnol CM, Marck PB. Carga de trabalho da equipe de enfermagem e segurança do paciente - estudo com método misto na abordagem ecológica restaurativa. Rev Lat Am Enfermagem 2013; 21(n. esp.):146-154.
27. Brasil. Emenda Constitucional no 95, de 15 de dezembro de 2016. Altera o Ato das Disposições Constitucionais Transitórias, para instituir o Novo Regime Fiscal, e dá outras providências. Diário Oficial da União 2016; $15 \mathrm{dez}$.

28. Gaulejac V. Gestão como doença social. São Paulo: Ideias \& Letras; 2007.

29. Pereira RCA, Rivera FJU, Artmann E. The multidisciplinary work in the family health strategy: a study on ways of teams. Interface (Botucatu) 2013; 17(45):327340.

30. Fernandes HN, Thofehrn MB, Porto AR, Amestoy SC, Jacondino MB, Soares MR. Relacionamento interpessoal no trabalho da equipe multiprofissional de uma unidade de saúde da família. Rev Pesqui Cuid Fundam 2015; 7(1):1915-1926.

31. Santana JS, Azevedo TL, Reichert APS, Medeiros AL, Soares MJGO. Núcleo de Apoio à Saúde da Família: atuação da equipe junto à Estratégia Saúde da Família. Rev Pesqui Cuid Fundam 2015; 7(2):2362-2371.

32. Santos ROM, Romano VF, Engstrom EM. Vínculo longitudinal na Saúde da Família: construção fundamentada no modelo de atenção, práticas interpessoais e organização dos serviços. Physis [periódico na Internet]. 2018 [acessado $2019 \mathrm{Fev} 25$ ]; 28(2):e280206. Disponível em: http://dx.doi.org/10.1590/s0103-7331 2018280206

33. Souza MG, Mandu ENT, Elias AN. Percepções de enfermeiros sobre seu trabalho na Estratégia Saúde da Família. Texto Contexto Enferm 2013; 22(3):772-779.

Artigo apresentado em 25/02/2019

Aprovado em 20/08/2019

Versão final apresentada em 02/10/2019 